\title{
High-temperature spin dynamics in the Heisenberg chain: Magnon propagation and emerging Kardar-Parisi-Zhang scaling in the zero-magnetization limit
}

\author{
Felix Weiner, ${ }^{1}$ Peter Schmitteckert $\odot,{ }^{2}$ Soumya Bera $\odot,{ }^{3}$ and Ferdinand Evers ${ }^{1}$ \\ ${ }^{1}$ Institute of Theoretical Physics, University of Regensburg, D-93040 Regensburg, Germany \\ ${ }^{2}$ HQS Quantum Simulations GmbH, 76131 Karlsruhe, Germany \\ ${ }^{3}$ Department of Physics, Indian Institute of Technology Bombay, Mumbai 400076, India
}

(Received 13 September 2019; revised manuscript received 6 December 2019; published 13 January 2020)

\begin{abstract}
The large-scale dynamics of quantum integrable systems is often dominated by ballistic modes due to the existence of stable quasiparticles. We here consider as an archetypical example for such a system the spin- $\frac{1}{2}$ XXX Heisenberg chain that features magnons and their bound states. An interesting question, which we here investigate numerically, arises with respect to the fate of ballistic modes at finite temperatures in the limit of zero magnetization $m=0$. At a finite magnetization density $m$, the spin-autocorrelation function $\Pi(x, t)$ (at high temperatures) typically exhibits a trimodal behavior with left- and right-moving quasiparticle modes and a broad center peak with slower dynamics. The broadening of the fastest propagating modes exhibits a subdiffusive $t^{1 / 3}$ scaling at large magnetization densities $m \rightarrow \frac{1}{2}$, familiar from noninteracting models; it crosses over into a diffusive scaling $t^{1 / 2}$ upon decreasing the magnetization to smaller values. The behavior of the center peak appears to exhibit a crossover from transient superdiffusion to ballistic relaxation at long times. In the limit $m \rightarrow 0$, the weight carried by the propagating peaks tends to zero; the residual dynamics is carried only by the central peak; it is sub-ballistic and characterized by a dynamical exponent $z$ close to the value $\frac{3}{2}$ familiar from Kardar-ParisiZhang (KPZ) scaling. We confirm that, employing elaborate finite-time extrapolations, that the spatial scaling of the correlator $\Pi$ is in excellent agreement with KPZ-type behavior and analyze the corresponding corrections.
\end{abstract}

DOI: 10.1103/PhysRevB.101.045115

\section{INTRODUCTION}

Interaction effects in strictly one-dimensional quantum systems tend to be strong. This is, roughly speaking, because the dimensional reduction weakens the efficiency of screening and makes it difficult for two excitations approaching each other to avoid a collision. The overall reduction of phase space for (few-body) scattering processes has one more interesting consequence: Classes of one-dimensional model systems (integrable) can be identified that carry an extensive amount of conserved quantum numbers and their thermodynamic properties can be interpreted in terms of effective particles [1].

When it comes to the hydrodynamic regime, it is well known that conservation laws tend to manifest in the analytical structures of kinetic coefficients. Therefore, it is an interesting endeavor to inquire into the hydrodynamics of fully integrable systems as has been done, recently.

Corresponding generalized hydrodynamic descriptions (GHD) have been proposed [2,3]. They feature kinetic equations for generalized phase-space distributions, akin to the theory of classical soliton gases [4,5]. Diffusive corrections and entropy production due to quasiparticle scattering have been incorporated recently [6-8]. Moreover, quantum hydrodynamics for one-dimensional systems at zero temperature developed earlier, ${ }^{1}$ formulated in terms of density and velocity

\footnotetext{
${ }^{1}$ The development of hydrodynamics for 1D systems is a field with a long history. We only cite one of the pioneering works, Ref. [43], and Refs. $[9,44]$ for a more recent overview.
}

fields, was shown to be reproduced by GHD in the corresponding limit [9]. In this work, we numerically investigate the spin dynamics in isotropic Heisenberg chains at high temperatures for varying total magnetization density $m$. In the limit $m \rightarrow-\frac{1}{2}$, i.e., close to the ferromagnetic vacuum, only bare magnons contribute to the spin-autocorrelation function $\Pi(x, t)$, visible as a cone in Fig. 1. The front of the cone exhibits a time window of subdiffusive broadening reflecting the dispersion of the quasiparticles (see Ref. [10] and references therein). The crossover time into the true asymptotic regime, which we find to be diffusive, consistent with Ref. [11], is expected to diverge in the limit $m \rightarrow-\frac{1}{2}$. Concomitantly, at intermediate $m$, additional ballistic modes can be identified corresponding to 2-magnon bound states.

An intriguing question arises about the fate of the dynamics in the limit of zero magnetization. In this case, it is understood that the quasiparticles effectively do not carry magnetization [12]. Consistently, we observe the weight of the propagating peaks in $\Pi(x, t)$ to disappear. At zero magnetization $m=0$, and elevated temperatures $T \rightarrow \infty$, a sub-ballistic dynamics of spin excitations takes over, as has been reported in a number of numerical studies dating back to [13]. However, it has long remained controversial whether a ballistic contribution to the spin dynamics, as measured by the Drude weight, exists (see Ref. [14] for an overview). Most recent analytical studies suggest that the Drude weight indeed vanishes for any finite $T>0[14,15]$. The peculiar residual dynamics has been identified as superdiffusive relaxation with a dynamical exponent close to $z \approx \frac{3}{2}$ [16-20]. Consistent with numerical observations, it was confirmed analytically that the spin-diffusion 

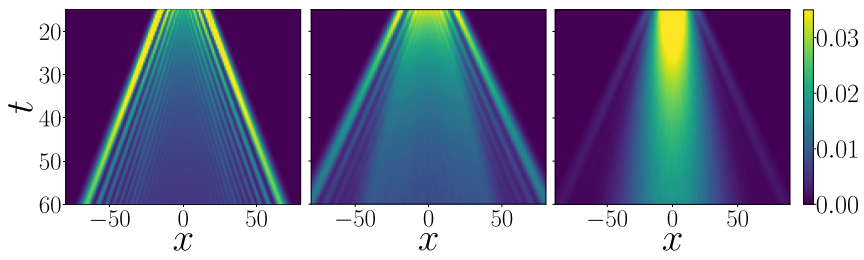

FIG. 1. Spin-autocorrelation function $\Pi(x, t)$ for totalmagnetization density $m \approx-0.48,-0.38,-0.17$ (left, center, right) and $m=-0.17$ (right). Ballistic propagation manifests itself in the "light-cone" structures. At small magnetization a broad center peak is seen that develops a critical, KPZ-type dynamics in the limit $m \rightarrow 0$. Data have been calculated as explained in the Sec. II B.

coefficient diverges [12]. Recently, Ref. [21] showed numerically that not only the exponent $z$, but also the spatial shape of the spin-spin autocorrelation function is in agreement with Kardar-Parisi-Zhang (KPZ) scaling. We confirm this result and carefully analyze the corrections to KPZ scaling, which we find to be of the form $t^{-y}, y \approx \frac{1}{3}$. Our calculations for the high-temperature spin autocorrelator follow a protocol pioneered in Ref. [17], employing standard matrix product operator (MPO) techniques. We observe that the magnetization dynamics with bond dimensions $\chi \leqslant 1000$ exhibits unphysical fluctuations for times $t \gtrsim 30$, which are short in comparison to the scaling limit. Remarkably, the qualitative characteristics of the long-time limit, such as the dynamical exponent and the KPZ scaling, appear to be rather forgiving in the sense that it emerges after removing fluctuations by performing running averages (see Ref. [21]).

\section{MODEL AND METHOD}

\section{A. Model and observable}

The Hamiltonian of the XXZ Heisenberg chain is given by

$$
\hat{H}=J \sum_{x=-\frac{L}{2}}^{\frac{L}{2}-1}\left[\hat{S}_{x}^{x} \hat{S}_{x+1}^{x}+\hat{S}_{x}^{y} \hat{S}_{x+1}^{y}+\Delta \hat{S}_{x}^{z} \hat{S}_{x+1}^{z}\right] .
$$

We choose $J=1$ as the unit of energy and concentrate on $\Delta=1 .^{2}$ The total $z$ component of spin $\hat{M}=\sum_{x} \hat{S}_{x}^{z}$ is conserved, i.e., commutes with $\hat{H}$. The length of the chain is chosen $L \geqslant 200$ such that, on the timescales shown, the boundaries do not affect the results of this work.

Our observable is the spin dynamics by means of the equilibrium $S^{z}$ correlation function:

$$
\Pi(x, t)=\mathcal{N}\left(\left\langle\hat{S}_{x}^{z}(t) \hat{S}_{0}^{z}\right\rangle_{h}-\left\langle\hat{S}_{0}^{z}\right\rangle_{h}^{2}\right)
$$

with $\hat{S}_{x}^{z}(t)=e^{i \hat{H} t} \hat{S}_{x}^{z} e^{-i \hat{H} t}$. Averages are taken with respect to an infinite-temperature ensemble

$$
\langle\hat{X}\rangle_{h}=\frac{\operatorname{Tr}\left[e^{-h \hat{M}} \hat{X}\right]}{\operatorname{Tr} e^{-h \hat{M}}},
$$

\footnotetext{
${ }^{2}$ The sign of $\Delta$ is insignificant for the observables studied in this work.
}

where $h$ controls the average magnetization $\langle\hat{M}\rangle_{h}=$ $\frac{L}{2} \tanh \left(\frac{h}{2}\right)$. The prefactor in (2) is time independent and normalizes the correlator: $\sum_{x} \Pi(x, t)=1$.

Instead of directly evaluating $\Pi(x, t)$, we adopt the simulation protocol suggested in [17] and compute the time evolution of a nonequilibrium initial state

$$
\hat{\rho}_{0} \sim \exp \left(-h \hat{M}-\sum_{x} \mu_{x} \hat{S}_{x}^{z}\right)
$$

corresponding to a high-temperature state with varying $M$ density. The initial spin profile has a "domain wall" shape:

$$
\mu_{x}= \begin{cases}+\mu, & x>0 \\ -\mu, & x \leqslant 0\end{cases}
$$

Then, in the limit of small $\mu$, the nonequilibrium spin densities can be related to the equilibrium correlator $\Pi(x, t)$ :

$$
\partial_{x} \operatorname{Tr}\left[\rho_{0} \hat{S}_{x}^{z}(t)\right] \approx \mu\left(\left(S_{x}^{z}(t) S_{0}^{z}\right\rangle_{h}-\left\langle S_{0}^{z}\right\rangle_{h}^{2}\right)+O\left(\mu^{2}\right) .
$$

The spatial derivative is evaluated numerically. While such a linear-response relation is easily seen to hold for continuous $x$, an exact relation for the lattice model was derived in Ref. [21]. We chose $\mu=0.001$ in the numerical simulations.

\section{B. Method}

Time evolution $\hat{\rho}(t)=e^{-i \hat{H} t} \hat{\rho}(t=0) e^{i \hat{H} t}$ is carried out using a matrix product decomposition of (4) (controlled by the maximum bond dimension $\chi$ ) and conventional Trotter decomposition (controlled by the time increment $\Delta t$ ) of the Liouvillian superoperator $\mathcal{L}$ corresponding to the Hamiltonian (1): $\mathcal{L} \hat{\rho} \equiv[\hat{H}, \rho]$. Conservation of $\hat{S}^{z}$ implies a blockdiagonal structure of $\hat{\rho}$, which is exploited in order to speed up the calculations. As these are standard techniques used in the field, further details are delegated to Appendix A. The calculations were performed using the ITensor library [22].

We offer a remark concerning the convergence with bond dimenion $\chi$. Quite generally, the convergence properties with respect to $\chi$ are far from universal: depending on the system (i.e., the Hamiltonian), the initial state, and certain computational details, convergence of a given observable can be reached at significantly different $\chi$ values. Indeed, a remarkable observation was made in [17]: Evolving initial states of type (4), the authors could obtain results for the spin and current densities that are roughly independent on $\chi$ up to long times $t \lesssim 150$. This exceeds simulation times reported for direct evaluation of correlation functions by almost an order of magnitude (see, e.g., Refs. [23,24] for an analysis of convergence properties).

While our results fully confirm the qualitative conclusions of $[17,21]$, we do observe corrections upon increasing $\chi$. For example, the diffusion constant at $\Delta=2$ is observed to keep increasing with $\chi>1000$ where its value has increased to $D \geqslant 0.63$ as opposed to $D \approx 0.4$ reported in [17] (see Appendix B). Based on the impression that results may still exhibit a significant dependence on the bond dimension, the convergence of dynamical properties with $\chi$ will receive 


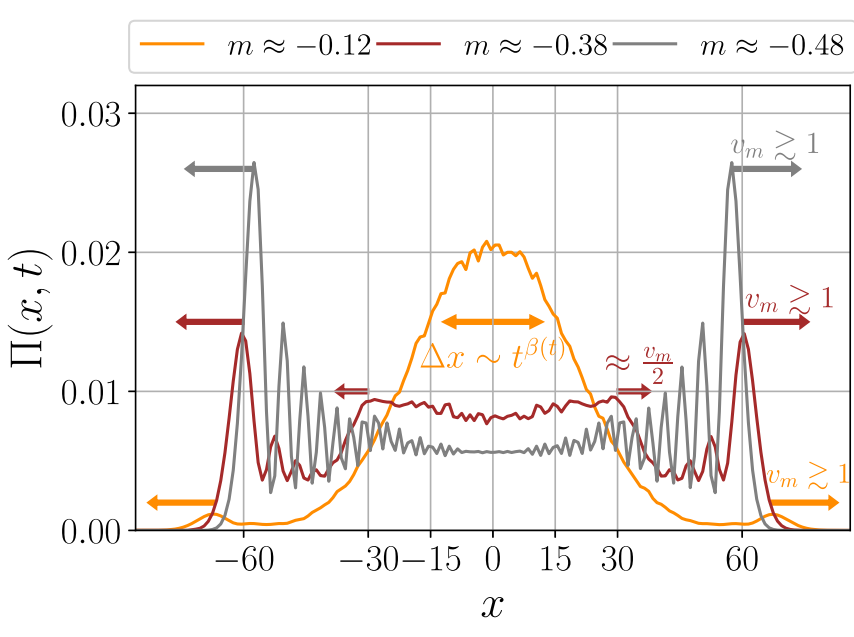

FIG. 2. Spin density profiles at time $t=60$ for varying magnetization density $m$. The outer peaks at $|x| \approx v_{m} t \approx 60$ correspond to propagating magnonlike excitations with a velocity $v_{m} \gtrsim 1$, which is slightly renormalized with increasing $m$. For $m=-0.38$, distinct peaks can be observed around $x \approx v_{m} t / 2 \approx 30$, which we attribute to 2-magnon bound states. The center peak seen for $m=-0.12$ exhibits sub-ballistic broadening $\sim t^{\beta_{m}(t)}$ on the timescales shown here. In the limit of $m \rightarrow 0$, our results are consistent with an exponent of $\beta_{m=0}(t \rightarrow \infty)=\frac{2}{3}$ as observed in previous studies.

a special attention below. An additional discussion of convergence behavior is given in Appendix A 3.

\section{RESULTS}

The form of the correlation function (2) is determined by the motion of the quasiparticles. We refer to Ref. [25] for a discussion of the analytic properties of this correlator. In the limit of $m=\langle\hat{M}\rangle_{h} / L \rightarrow-0.5$, the correlator $\Pi$ probes dynamics close to the fully polarized state. Excitations of this state are magnons and bound states of $n$ magnons with bare group velocity $v_{b} \sim \frac{J}{n}$ [1]. Due to the integrability of the model, quasiparticles remain stable even for $|m|<0.5$ and give rise to ballistic modes in the spin dynamics, which are observed in $\Pi(x, t)$ as a set of propagating peaks. The spatial dependence of $\Pi$ at fixed time for varying $m$ is illustrated in Fig. 2: At strong magnetization $|m| \rightarrow 0.5$, only magnons contribute to $\Pi$, which manifest themselves as a sequence of left- and right-moving peaks with velocities $\pm v_{m}$. The evolution of these peaks and their dependence on $m$ is analyzed in Sec. III A. At intermediate $|m|$ we can also identify another pair of distinct peaks in $\Pi$, which move with a slower velocity that is given by roughly half the magnon velocity (as indicated by the arrows in the figure). Therefore, they can be associated with 2-magnon bound states.

Upon further decreasing $|m|$, only a single propagating peak in $\Pi$ is left; the remaining weight is carried by a broad peak centered around $x=0$, at least on the timescales studied here. This peak exhibits anomalous KPZ scaling at vanishing total magnetization $m=0$, which we analyze in Sec. III B. The behavior of the center peak for finite $m$ is discussed in Sec. III C.

\section{A. Finite magnetization: Magnon modes}

\section{Shape of the magnon peak}

In the limit $m \rightarrow-0.5$, the correlator $\Pi(x, t)$ probes spin dynamics close to the fully polarized state, which is the (grand-canonical) ground state of the ferromagnetic Heisenberg chain. The fastest excitations of this state are free magnons with bare dispersion $\epsilon(k) \sim-J \cos (k)$ and a maximum group velocity of $v=J(=1)$.

In the context of noninteracting models, it is well known that nonlinearity of the free quasiparticle dispersion gives rise to a peculiar scale invariance of density profiles close to the "light cone" [26,27], i.e., for $x= \pm v t$ (where $v$ denotes the velocity of the fastest quasiparticle mode). More specifically, the broadening of the ballistic front is given by a subdiffusive power law $t^{1 / 3}$. Recently, attempts have been made to interpret these findings in the context of GHD [10,28]. We would like to stress that the exponents arising here, while similar to the KPZ exponents, are believed to have a different origin as the dynamical exponent $z$ discussed in Sec. III B 1 below. The latter is interpreted as a consequence of interactions between quasiparticles.

In Fig. 3(a) we show that, for large magnetization ( $m \approx-0.5$ ), П appears to exhibit the sub-diffusive scaling close to the light-cone, as in the non-interacting case:

$$
\Pi(x, t) \sim \frac{1}{t^{2 / 3}} F\left(\frac{x \pm v_{m} t}{t^{1 / 3}}\right)
$$

for $x \sim v_{m} t$, where the $F(y)=2^{2 / 3} \mathrm{Ai}^{2}\left(2^{1 / 3} y\right)$ with $\mathrm{Ai}(y)$ denoting the Airy function [26]. In order to achieve a collapse of our numerical data for different times, we need to account for a small renormalization of the bare magnon velocity (the actual velocity $v_{m}$ is taken as a fit parameter here).

The question of whether the $t^{1 / 3}$ scaling survives in the presence of interactions has been discussed in recent works $[10,11,28]$. In Ref. [28], it was shown numerically to occur for any values of $\Delta$ if the initial state is given by a polarized product state, consistent with our findings for the spin correlator. On the other hand, for more generic nonequilibrium situations, it is expected that diffusive dynamics [8] will eventually dominate over the dispersive $t^{1 / 3}$ scaling in the long-time limit. Numerical evidence for such a diffusive scaling of spin profiles close to the light cone was given in Ref. [11] in the regime $\Delta<1$. In the following, we study the crossover in more detail, as a function of both magnetization and time.

Upon decreasing $|m|$, going away from the fully polarized limit, we observe that the features of $F(y)$, as given in Eq. (7), are increasingly washed out. We indeed find that, for small enough $|m|$, the broadening of the magnon peak appears to follow a diffusive $t^{1 / 2}$ scaling at variance with the subdiffusive $t^{1 / 3}$ scaling observed for $|m| \rightarrow 0.5$ [see Fig. 3(c)]. From the reasoning above, one would expect that the width of the fastest propagating peak $\sigma_{\text {mag }}$ can be described by $\sigma_{\text {mag }}^{2}(t)=D_{\text {mag }} t+$ $(\kappa t)^{2 / 3}$. The latter prefactor $\kappa$ can be determined from our data in the limit $|m| \rightarrow \frac{1}{2}$ [see Fig. 3(a)], which yields $\kappa \approx 0.18$. Stipulating that $\kappa$ is independent of the magnetization $m$, we define a crossover timescale

$$
t_{c} \sim \frac{\kappa^{2}}{D_{\mathrm{mag}}^{3}} \approx \frac{0.03}{D_{\mathrm{mag}}^{3}},
$$




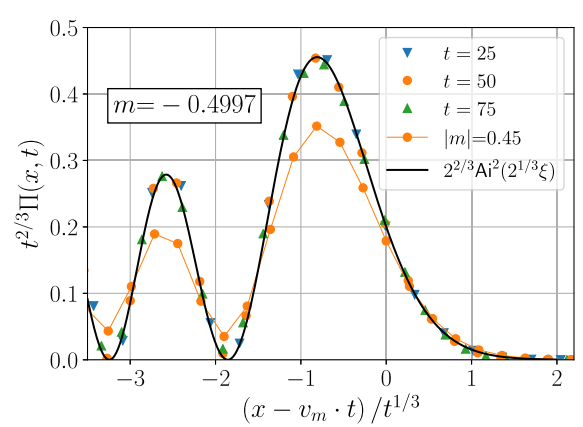

(a)

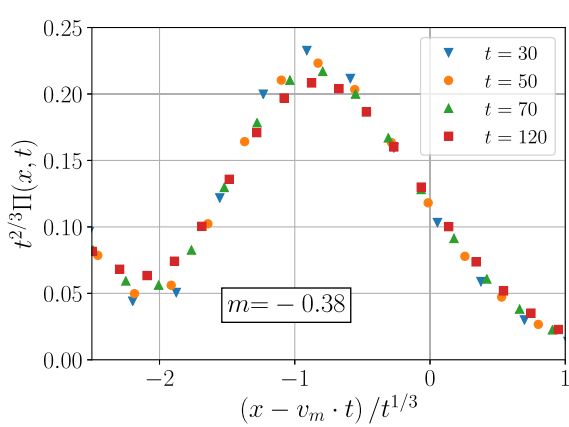

(b)

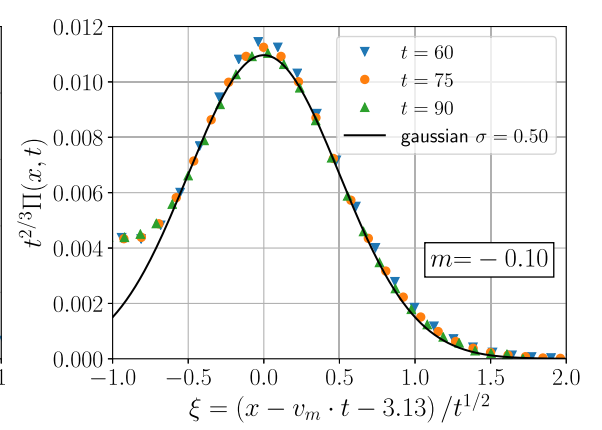

(c)

FIG. 3. Rescaled correlator close to the light cone $x=v_{m} t$ for different values of $m$. The figures illustrate the evolution of the magnon peak as a function of magnetization $m$ and time $t$. (a) In the strongly magnetized case $m \approx-0.5$, numerical results are compared with the exact scaling function for noninteracting systems [26]. We also show data for a slightly smaller value of $|m|$, which illustrates how the profile is smeared out away from the fully polarized limit. (b) For an intermediate value of the magnetization $|m| \approx 0.38$, we find that the scaling collapse, as observed in Fig. 3(a), holds only approximately at relatively short times. In order to illustrate the deviations, we also show data for $t=120$. (c) At $m \approx-0.1$, the numerical data can be described by a Gaussian close to the peak, i.e., around $\xi=0$. Note the different rescaling of the spatial coordinate as compared to Figs. 3(a) and 3(b). In the weakly magnetized limit, we found that a diffusive rescaling of the spatial coordinate yields a better collapse of the numerical data (see discussion in the main text).

such that for $t \ll t_{c}$ the broadening is subdiffusive $\sigma_{\mathrm{mag}} \sim t^{1 / 3}$, and for $t \gg t_{c}$ it is diffusive $\sigma_{\mathrm{mag}} \sim t^{1 / 2}$. For small and intermediate values of $|m|$, estimates for the prefactor $D_{\text {mag }}$ can be obtained from numerical data, e.g., from Gaussian fits as seen in Fig. 3(c). In this manner, we obtain the estimates for $t_{c}$ shown in Fig. 4. In the strongly magnetized limit, the diffusion constant associated with the broadening $D_{\text {mag }}$ is expected to be determined by the magnon occupation factor $\theta_{1}$ only $[12,19]$ :

$$
D_{\text {mag }} \sim \theta_{1}\left(1-\theta_{1}\right) \stackrel{|m| \rightarrow 1 / 2}{\approx} \theta_{1}=\left(1 / 4-m^{2}\right)
$$

while contributions from bound states are suppressed. Indeed, we find that our numerical results for $t_{c}$ are consistent with a divergence of the form $\sim\left(1 / 4-m^{2}\right)^{-3}$ upon approaching the fully magnetized limit $m=\frac{1}{2}$ (see Fig. 4).

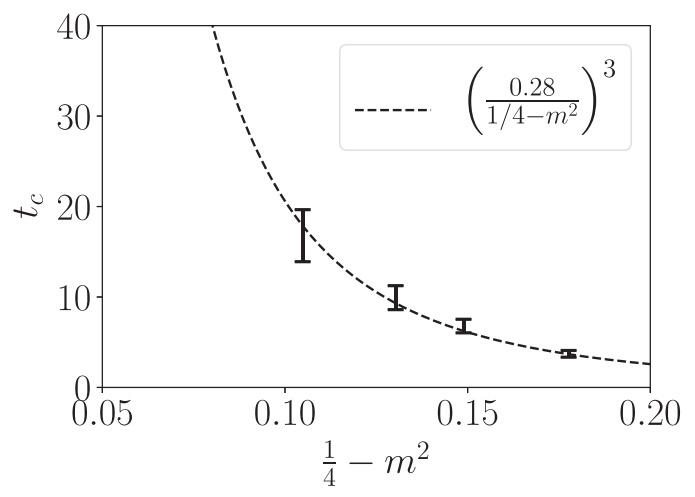

FIG. 4. Estimate for the crossover timescale that separates the diffusive $\left(t>t_{c}\right)$ from the subdiffusive $\left(t<t_{c}\right)$ growth of the width of the fastest propagating peak. The crosses indicate numerical results, while the dashed line is a conjecture based on Eq. (9). Error bars reflect the residual time dependence of the numerical values obtained for $D_{\text {mag }}$.

\section{Velocity}

Tracking the outermost peak appears to be the simplest scheme for extracting renormalized magnon velocities. We achieve this by fitting the numerical data to a Gaussian close to the peak and then obtain the velocity via linear regression. The results are shown in Fig. 5. For large magnetization $|m|$, however, such a scheme does not yield accurate results on the timescales $t \leqslant 100$ studied in this work. The reason for this failure becomes obvious from the scaling shown in Fig. 3(a): $\xi=0$ does not correspond to the position of the peak but rather to a different point at larger $\xi$ (corresponding to the turning point of the Airy function). Therefore, the position of the peak $x_{p}(t)$ exhibits a subleading term $x_{p}=v_{m} t+$ const $\times t^{-2 / 3}+\cdots$ at short times, when the subdiffusive scaling still holds approximately. We also show velocities obtained from fitting the profile to the function $F(y)$ [see Eq. (7)] at large $|m|$. The thus obtained values linearly extrapolate to the correct bare magnon velocity: $v_{m} \approx 1.0+0.43(|m|-1 / 2)$

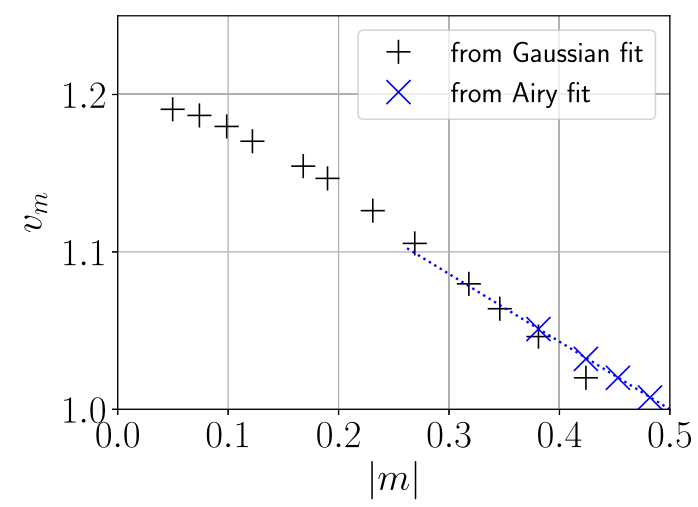

FIG. 5. Renormalized velocities of the "magnon peak" as a function of the magnetization density $m$. The dashed line corresponds to a linear fit of the blue data points, which have been obtained obtained from fitting the ballistic peaks to the scaling function (7) [cf. Fig. 3(a)]. 


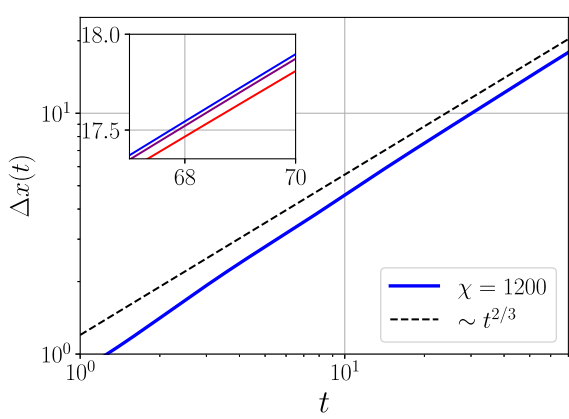

(a)

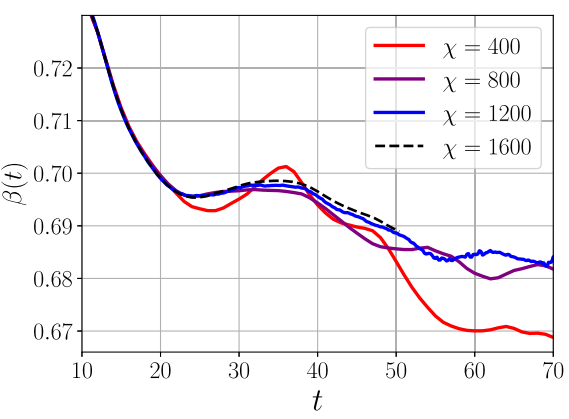

(b)

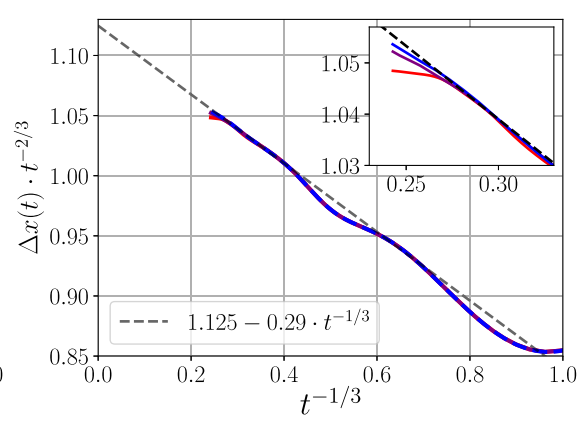

(c)

FIG. 6. Characteristics of the spin dynamics computed from $\Pi(x, t)$ at zero magnetization $m=0$. (a) Time dependence of the width $\Delta x$ of the correlator. The black line serves as a guide to the eye, indicating a power law $t^{1 / z}$ corresponding to the KPZ exponent $z=\frac{3}{2}$. The inset highlights the dependence on the maximum bond dimension $\chi$, which is not visible in the main plot. (b) The effective, time-dependent exponent $\beta(t)$ [see Eq. (11)] highlighting deviations from true power-law behavior and significant residual dependency on the bond dimension $\chi$. (c) The graph suggests that corrections to the power-law behavior can be described by a subleading term $\sim t^{1 / 3}$. The offset and slope obtained from the fit (dashed black line) are discussed in the main text.

for $m \gtrsim-1 / 2$. In the opposite limit $m \rightarrow 0$, the renormalized magnon velocity appears to approach a value of $v_{m=0} \approx 1.2$, which is consistent with the value for the "Lieb-Robinson" velocity at vanishing magnetization that was obtained in [12] (see Fig. 1, inset, of that reference).

\section{B. Spin profiles at $m=0$ : KPZ scaling}

A recent numerical work [21] studied the high-temperature spin correlator (2) in the isotropic Heisenberg chain at vanishing total magnetization, i.e., $m=0$. Interestingly, the authors found that the spatial profile is given by scaling functions of the KPZ universality class, consistent with the dynamical exponent $z=\frac{3}{2}$ observed earlier [16,17]. In this section we confirm these observations by carefully analyzing transients and corrections to scaling, as well as the dependence of the numerical results on the bond dimension.

The KPZ equation was initially suggested as a description of universal properties of surface growth [29]. The closely related stochastic Burger's equation appears as a hydrodynamic limit in many classical many-body systems in one dimension (see, e.g., Ref. [30]). Manifestations of KPZ universality in quantum systems are subject to ongoing research (see Refs. [31-34] for works outside of the present context). It should be noted, however, that a theoretical understanding of why KPZ universality emerges in the integrable XXX chain is still lacking. Some aspects of the superdiffusive dynamics have been captured by a kinetic theory [19]. Furthermore, numerical studies have provided insight regarding the relevant conservation laws: A recent study indicates that integrability is indeed a crucial ingredient in order to observe a dynamical exponent $z=\frac{3}{2}$ in spin chains; ${ }^{3}$ the relevancy of energy conservation is presently investigated [21].

\footnotetext{
${ }^{3}$ See Ref. [20]. It should be noted, however, that previous studies found superdiffusive dynamics also in the nonintegrable spin-1 Heisenberg chain $[38,45]$ at high temperatures.
}

\section{Time evolution of $\Delta x(t)$}

As ballistic contributions are absent at $m=0$, the dynamics of the center peak is characterized by the width

$$
\Delta x(t)=\left(\sum_{x} x^{2} \Pi(x, t)\right)^{1 / 2} .
$$

This quantity can be interpreted as the root-mean-squared displacement of an excess spin density initially localized at the origin $x=0$. The corresponding numerical data are displayed in Fig. 6(a), exhibiting an approximate power law $t^{1 / z}$ with dynamical exponent $z \approx 1.5$. $z$ being close to $\frac{3}{2}$ has been observed before $[16,17]$ and was giving a motivation to inquire into the possibility of KPZ dynamics.

Convergence of effective exponent function.. In order to highlight the deviations from a true power-law behavior as well as the dependence on the bond dimension $\chi$, we introduce the effective exponent

$$
\beta(t)=\frac{d \log \Delta x(t)}{d \log (t)} .
$$

Results are shown in Fig. 6(b). While saturation of $\beta(t)$ near a value of $\frac{2}{3}$ is observed at relatively small $\chi$, deviations grow at better $\chi$ values; concomitantly, the "noise" on $\beta(t)$ seen in Fig. 6(b) diminishes. Strictly speaking, the asymptotic value $\beta(t \rightarrow \infty)$ is not reliably obtained from the data without further analysis.

Corrections to scaling.. To obtain a reliable estimate of $\beta(t \rightarrow \infty)$ we analyze the transients, i.e., preasymptotic corrections. Our data suggest the following functional form:

$$
\Delta x \approx a t^{2 / 3}\left(1+b t^{-1 / 3}\right)
$$

[see Fig. 6(c)]. By extrapolation of the numerical data (as indicated in the figure), we obtain $\Delta x \approx 1.125 t^{2 / 3}$ for the leading term. The numerical value of the prefactor will be discussed below. While the exponent of the subleading term in Eq. (12) is difficult to determine with certainty, an expansion of $\Delta x$ in powers of $t^{1 / 3}$ appears natural. 


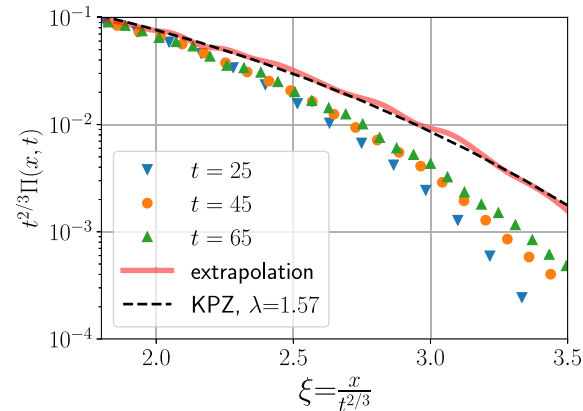

(a)

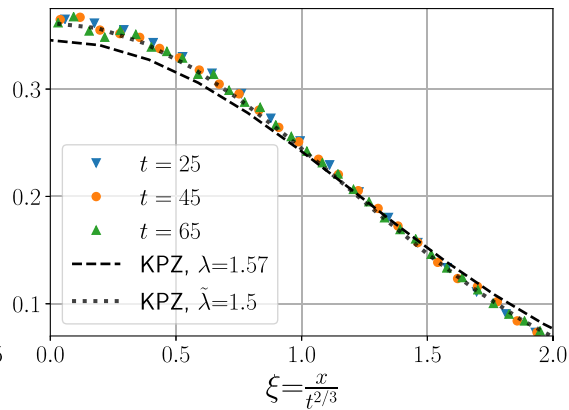

(b)

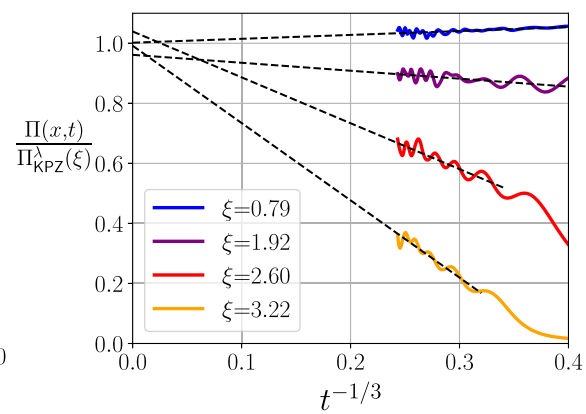

(c)

FIG. 7. (a) Numerical data for the tails of $\Pi(x, t)$, rescaled assuming a dynamical exponent of $z=\frac{3}{2}$, is compared to the KPZ prediction $\lambda^{-1} f_{\mathrm{KPZ}}\left(\frac{x}{\lambda t^{2 / 3}}\right)$ taken from [36]. Numerical results clearly exhibit a residual time dependence in the tails of $\Pi$. The parameter $\lambda=1.57$ has been chosen in order to be consistent with our extrapolation of $\Delta x(t)$. The important point of this figure is that the red line, corresponding to an extrapolation with respect to time, agrees very well with the KPZ prediction. (b) The center of the correlation function $\Pi$ can be fitted by the KPZ scaling function with $\lambda=1.5$. The value of $\lambda$ obtained from such a fit is still time dependent on the timescales shown here (see discussion in the main text). (c) Illustration of the extrapolation scheme, which gives rise to the values of the red line shown in Fig. 7(a). Extrapolation is carried out for a fixed $\xi=\frac{x}{t^{2 / 3}}$ (after interpolating numerical data) presuming that corrections to scaling follow Eq. (15). The irregular oscillations in the data, which are observed at longer times, are a signature of truncation errors (see Appendix A 3).

\section{Spatial profile: Comparison with KPZ scaling}

We now turn to the analysis of how $\Pi(x, t)$ depends on the spatial coordinate $x$ and compare it with the relevant KPZ scaling function $f_{\mathrm{KPZ}}(x) .{ }^{4}$ Exact results for $f_{\mathrm{KPZ}}$ were obtained in Ref. [35] and its numerical values have been tabulated [36]. $f_{\mathrm{KPZ}}$ resembles a Gaussian for small arguments, but it exhibits faster decay in the tails: $f_{\mathrm{KPZ}}(y) \sim \exp \left(-C \times|y|^{3}\right)$ for $|y| \gg 1$ with $C \approx 0.3$.

KPZ universality would imply the following scaling form of the spin correlator:

$$
\Pi(x, t)=\frac{1}{\lambda t^{1 / z}} f_{\mathrm{KPZ}}\left(\frac{x}{\lambda t^{1 / z}}\right) \equiv \Pi_{\mathrm{KPZ}}^{\lambda}\left(\frac{x}{t^{1 / z}}\right)
$$

with $z=\frac{3}{2}$. Our results for $\Pi(x, t)$ are shown in Fig. 7 as a function of the scaling variable $\xi=\frac{x}{t^{2 / 3}}$. Before discussing the spatial dependence of the correlator, we recall our earlier result suggesting $\Delta x(t) / t^{2 / 3} \approx 1.125$ in the long-time limit [see Eq. (12)]. Presuming KPZ scaling, we can relate the asymptotic time dependence of $\Delta x$ to the parameter $\lambda$ via

$$
\frac{\Delta x(t)}{t^{2 / 3}} \stackrel{t \rightarrow \infty}{\longrightarrow} \lambda\left(\int d y y^{2} f_{\mathrm{KPZ}}(y)\right)^{1 / 2} \approx 0.715 \lambda,
$$

which implies $\lambda \approx 1.125 / 0.715 \approx 1.57$. The corresponding prediction for the correlator $\Pi(x, t)$ together with the numerical data is shown in Fig. 7(b). We observe that the spatial shape based on KPZ scaling $\Pi_{\mathrm{KPZ}}^{\lambda=1.57}$ deviates from the numerical data for the relatively short times $t=35,45,65$ shown here. However, the results for $t^{2 / 3} \Pi(\xi)$ still exhibit a time dependence, which is most easily seen in the tails of the correlator [see Fig 7(a)]. Consistent with the analysis above, we suggest that such finite-time corrections vanish as $t^{-y}$

\footnotetext{
${ }^{4}$ In the original context, $f_{\mathrm{KPZ}}$ determines the asymptotic shape of the correlation function $\left\langle v(x, t) v\left(x^{\prime}, t^{\prime}\right)\right\rangle$, where $v(x, t)$ denotes a solution of the stochastic Burger's equation and $\langle\ldots\rangle$ averaging with respect to realizations of the noise.
}

with $y=\frac{1}{3}$ :

$$
\Pi(x, t)=\Pi_{\mathrm{KPZ}}^{\lambda}(\xi)\left(1+g(\xi) t^{-1 / 3}\right),
$$

which indeed yields an accurate and consistent description of the numerical results, as can be seen from Fig. 7(c). We note that the numerical data for $\xi \lesssim 2$, on the timescales shown in Fig. 7(b), agree well with a KPZ scaling corresponding to an effective value $\tilde{\lambda} \approx 1.5$. At even longer times this effective scale $\tilde{\lambda}$ will eventually converge to $\lambda$ proper. The small deviation seen in Fig. 7(b) is not inconsistent with our analysis, but rather a trivial consequence of the normalization of $\Pi(x, t) .^{5}$

Alternative interpretations based on a subleading power law with a different exponent $y>\frac{1}{3}$, as discussed in Appendix $\mathrm{C}$, are possible. On the other hand, it seems unlikely that the corrections decay even slower than $t^{-1 / 3}$. Therefore, our estimate $\lambda=1.57$ could be considered an upper bound for the possible values of $\lambda$ that are still consistent with the numerics.

The authors of Ref. [21] conjectured that $\lambda$ is exactly given by $\frac{3}{2}$, based on their numerical results. This conjecture appears inconsistent with our analysis. However, we suspect that the employed bond dimensions are not chosen sufficiently large in order to properly capture the transients. In fact, our results indicate that smaller bond dimensions tend to underestimate corrections to scaling (see Fig. 6). It is also interesting to note that Ref. [37] reports a very similar value of $\lambda \approx 1.55$ for the integrable classical analog of the XXX chain at high temperatures.

\section{Return probability}

The "return probability" $\Pi(x=0, t)$ is a probe of the central peak. At zero magnetization $m=0$, this peak exhibits the

\footnotetext{
${ }^{5} \mathrm{~A}$ similar time dependence of $\lambda$ has been observed in numerical works demonstrating KPZ scaling in classical models (see Refs. [46,47]).
} 


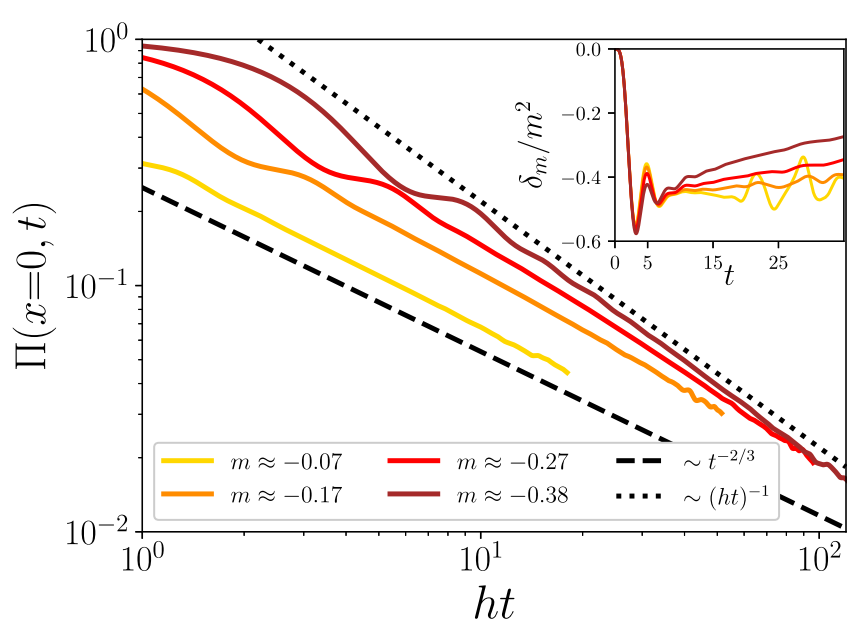

FIG. 8. Double-logarithmic plot of the return probability exhibiting the long-time, crossover behavior. The horizontal axis is rescaled in order to highlight the $\frac{1}{h t}$ behavior at long times. Black lines serve as guides to the eye. Inset: difference between the return probability $\Pi(x=0, t)$ at finite $m$ and $m=0$ scaled by $m^{2}$. The data (nearly) collapse at short times $t \lesssim 10$; at larger times a plateau develops for very small magnetizations $m(h)$. The fluctuations ("noise") seen in the small- $m$ data at larger times are expected to disappear in the limit of large $\chi(\chi=800$ was used here $)$.

anomalous KPZ scaling. It is interesting to inquire to what extent the anomalous scaling survives at finite $m$ and how the crossover $m \rightarrow 0$ occurs. This question has been addressed in a recent work [25] by means of kinetic theory as well as MPO numerics. In the following, we present an analysis of our numerical data and thereby confirm some of the conclusions of Ref. [25].

Short and intermediate times. As a measure for the impact of finite $m$, we define

$$
\delta_{m}(t)=\left.\Pi(0, t)\right|_{m}-\left.\Pi(0, t)\right|_{m=0} .
$$

At $|m|$ relatively small, one expects a low impact only, as long as times are not too large, so assuming analyticity: $\delta_{m}(t) \propto m^{2}$. As shown in Fig. 8 (inset), this is consistent with the simulation data in the window $0<t \lesssim 10$. The interpretation is straightforward: outmoving magnon modes carry spectral weight away from the center peak. At larger times and at $|m|$ small enough, we observe a plateau in $\delta_{m}(t)$, i.e., in this time window $\left.\Pi(x=0, t)\right|_{m}=\left.\Pi(x=0, t)\right|_{m=0}-$ $C \times m^{2}$ with $C \approx 0.45$. These findings underline that the anomalous KPZ-type behavior appears on an intermediate timescale once the magnetization $|m|$ is small enough, as one would expect.

Long times. The time dependence of $\Pi(x=0, t)$ at longer times is displayed in Fig. 8. The horizontal axis is rescaled in order to highlight the expected ballistic behavior

$$
\left.\Pi(x=0, t)\right|_{m} \sim \frac{1}{h t}, h=2 \operatorname{arctanh}(2 m)
$$

which reflects that magnonic quasiparticles carry away a finite magnetization at $|m|>0$ [25]. The crossover from KPZ to ballistic behavior is expected at times $t^{-2 / 3} \sim(h t)^{-1}$, so $t^{*} \sim$ $h^{-3}$, consistent with [25]. At small values $h \ll 1$, the time $t^{*}$ is well outside of our observation window. In this time window, the data displayed in Fig. 8 are still consistent with an anomalous power law $\Pi(x=0, t) \sim t^{-2 / 3}$. We do not actually observe a proper crossover in our numerical results, which would require long simulation times at a relatively small $|\mathrm{m}|$ and, therefore, large bond dimensions $\chi$. We interpret the curvature of the data shown in Fig. 8 (on a doubly logarithmic scale), e.g., for $m \approx-0.17$, as an indication for the existence of such a crossover.

\section{SUMMARY AND OUTLOOK}

We have presented a comprehensive discussion of the $S^{z}$ autocorrelation function $\Pi(x, t)$ for the spin- $\frac{1}{2}$ XXX Heisenberg chain at high temperature and fixed magnetization density $m$. For any finite $|m|>0$, the correlator $\Pi$ exhibits leftand right-moving peaks that we attributed to magnon-type quasiparticles. The time dependence of the broadening associated with those peaks exhibits different behavior depending on $|m|$ : Near maximum magnetization $|m| \lesssim \frac{1}{2}$, the broadening follows a subdiffusive $t^{1 / 3}$ scaling within our window of observation times, which we assign to (cubic terms of) the bare quasiparticle dispersion. For weaker magnetization $|m| \ll \frac{1}{2}$ a long-time regime emerges with $t^{1 / 2}$ broadening that we loosely interpret as a signature of quasiparticle scattering. We interpret our results in terms of a transient behavior for $t<t_{c}$ following a $t^{1 / 3}$ law, which gives way to a $t^{1 / 2}$ law at long times $t>t_{c}$. Our results are consistent with a crossover timescale $t_{c} \sim\left(1 / 4-m^{2}\right)^{-3}$.

At small $|m|$, a broad center peak dominates $\Pi(x, t)$. The return probability $\Pi(0, t)$ characterizes the corresponding dynamics. Also here, we find different behavior depending on $|m|$ : ballistic decay $t^{-1}$ is observed for the larger values of $|m|$, presumably reflecting the loss of amplitude due to the outgoing quasiparticles. On the other hand, close to $|m|=0$, $\Pi(0, t)$ decays in a sub-ballistic fashion following a $t^{-2 / 3}$ behavior. Our results are consistent with a crossover time $t^{*} \sim h^{3}$ between the two regimes, in agreement with the prediction of Ref. [25].

At zero magnetization $m=0$, the propagating peaks are absent. The width of the correlator can be described by $\Delta x \approx$ $a t^{2 / 3}\left(1+b t^{-y}\right)$, with $a \approx 1.125, b \approx-0.26$, and $y=\frac{1}{3}$. Motivated by the $t^{2 / 3}$ phenomenology, recent numerical work has tested $\Pi(x, t)$ against KPZ scaling and indeed demonstrates matching with the KPZ scaling function [21]. We confirm this result after including finite-time corrections. At this point, it seems that a deeper understanding of why KPZ scaling emerges in this model still needs to be developed in future research. Such understanding appears even more relevant as recent works suggest that the KPZ behavior does not only occur in the spin- $\frac{1}{2}$ chain but in a large class of integrable systems [20,37,38].

\section{ACKNOWLEDGMENTS}

The authors acknowledge valuable discussions with $\mathrm{H}$. Spohn, A. Läuchli, and B. N. Narozhny and thank T. Prosen for a helpful correspondence on computational details. P.S. acknowledges support by ERC-StG-ThomaleTOPOLECTRICS-336012. S.B. acknowledges support from SERB-DST, India, through Ramanujan Fellowship Grant No. 


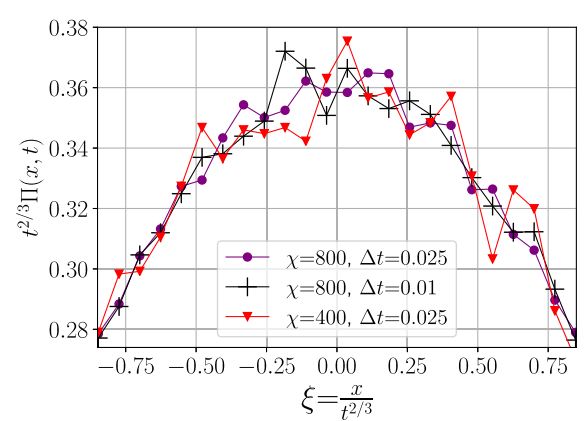

(a)

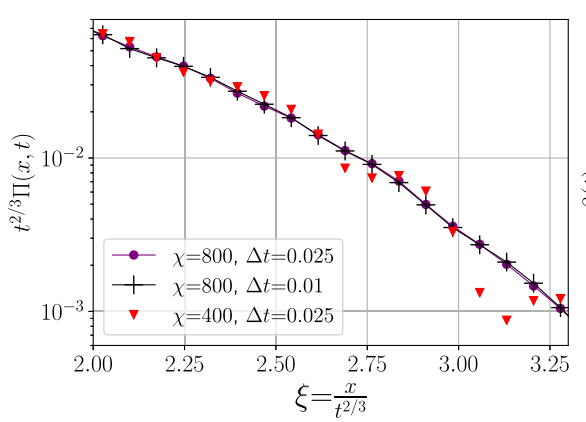

(b)

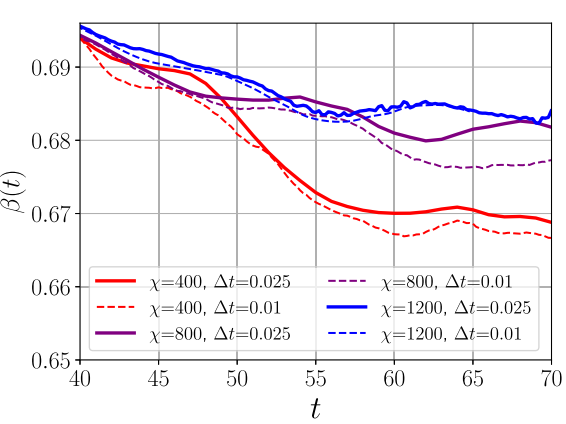

(c)

FIG. 9. (a), (b) Rescaled correlator $(h=0)$ at fixed time $t=50$ for various bond dimensions $\chi$ and Trotter time increments $\Delta t$. Truncation errors due to finite $\chi$ manifest themselves as unphysical spatial fluctuations, which are most pronounced near the center $x \sim 0$. At a given time, the tails are generally less affected by the truncation and are almost independent of $\Delta t$. (c) The effective exponent as shown in Fig. 6(b) together with additional data obtained by using a smaller Trotter increment $\Delta t=0.01$. Up to times $t \sim 60$, the data are not very sensitive to varying $\Delta t$.

SB/S2/RJN-128/2016, Early Career Research Award No. ECR/2018/000876, and MPG for funding through the Max Planck Partner Group at IITB. F.E. and F.W. acknowledge support by the DFG under Grants No. EV30/11-1, No. EV30/12-1, and No. SFB-1277, Project A03. We furthermore acknowledge assistance by I. Kondov and computing time on the supercomputer ForHLR funded by the Ministry of Science, Research and the Arts Baden-Württemberg and by the Federal Ministry of Education and Research.

\section{APPENDIX A: MATRIX PRODUCT STATE TECHNIQUES}

In this section, we briefly review the techniques employed to compute the time evolution of the initial state (4) and present an additional discussion of convergence properties. For details, we refer to reviews of the topic, e.g., Refs. [39,40].

\section{Mixed state representation}

The matrix product representation of any operator [a socalled matrix product operator (MPO)] is equivalent to a matrix product state (MPS) with an enlarged local Hilbert space [41]. We choose the standard basis in operator space as a local basis set: $|0\rangle\rangle=|\downarrow\rangle\langle\downarrow|| 1\rangle,\rangle=|\downarrow\rangle\langle\uparrow|| 2\rangle,\rangle=|\uparrow\rangle\langle\downarrow|| 3\rangle,\rangle=$ $|\uparrow\rangle\langle\uparrow|$. Then, a generic MPS representation (in operator space) of an operator $\hat{A}$ reads as

$$
\left.\hat{A} \widehat{=}|\hat{A}\rangle\rangle=\sum_{\{\Sigma\}} A_{1}^{\left[\Sigma_{1}\right]} A_{2}^{\left[\Sigma_{2}\right]} \cdots A_{L}^{\left[\Sigma_{L}\right]}|\{\Sigma\}\rangle\right\rangle,
$$

$$
\Sigma_{i} \in\{0,1,2,3\}
$$

where $A_{i}^{[\Sigma]}$ denote matrices of dimensions $\chi_{i} \times \chi_{i+1}, \chi_{i} \leqslant$ $\chi_{\max }$, and $\chi_{1, L}=1 . \chi_{\max }$ denotes the maximum bond dimension of the MPS. In practice, we do not represent the density matrix in MPS form but its square root. This enforces positivity of the physical density operator and it allows to write the expectation values of observables in the same form as for pure states:

$$
\operatorname{Tr}(\hat{\rho} \hat{O})=\operatorname{Tr}(\sqrt{\hat{\rho}} \hat{O} \sqrt{\hat{\rho}})=\langle\langle\sqrt{\hat{\rho}}|\mathcal{O}| \sqrt{\hat{\rho}}\rangle
$$

Here, $\mathcal{O}$ denotes a superoperator extension of $\hat{O}$ and the natural scalar product in operator space is given by the Frobenius product $\langle\langle\hat{A} \mid \hat{B}\rangle\rangle=\operatorname{Tr}\left(\hat{A}^{\dagger} \hat{B}\right)$.

\section{Time evolution}

The initial state (4) corresponds to a trivial MPO, i.e., it is a product state in operator space. As a close system is considered, its time evolution is governed by von Neumann equation $i \partial_{t} \hat{\rho}(t)=[\hat{H}, \hat{\rho}] \widehat{=} \mathcal{L}|\hat{\rho}(t)\rangle$, where $\mathcal{L}$ denotes the superoperator $\mathcal{L} \hat{O}=[\hat{H}, \hat{O}]$. Using this notation, we can introduce the analog of the time-evolution operator

$$
|\hat{\rho}(t)\rangle\rangle=\mathcal{U}(t)|\hat{\rho}(0)\rangle\rangle=\exp (-i \mathcal{L} t)|\hat{\rho}(0)\rangle\rangle .
$$

For models with nearest-neighbor terms only (as considered here), $\mathcal{L}$ can be written as

$$
\mathcal{L}=\sum_{x=1}^{L-1} \mathcal{L}_{x, x+1},
$$
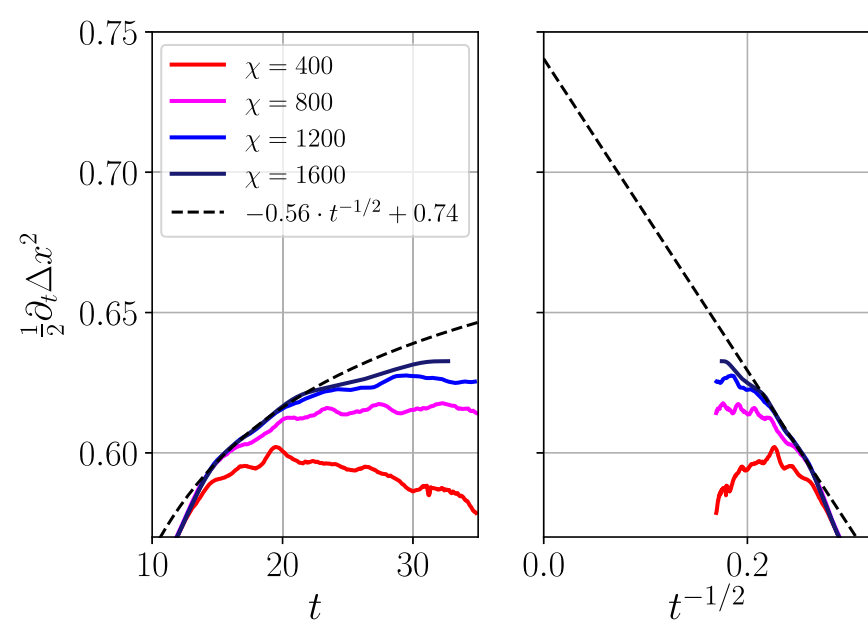

FIG. 10. Temporal derivative of $\Delta x^{2}(t)$ for various values of the maximum bond dimension at anisotropy $\Delta=2$. The dashed line corresponds to an extrapolation assuming that $\partial_{t} \Delta x^{2}=D+$ const $\times t^{-1 / 2}$. The extrapolated value $D \approx 0.74$ is consistent with the result of Ref. [8]. 

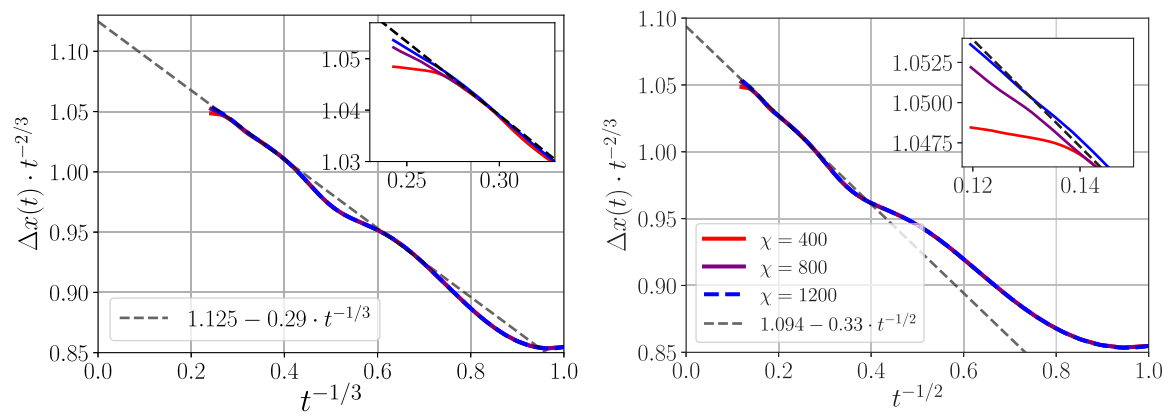

(a)

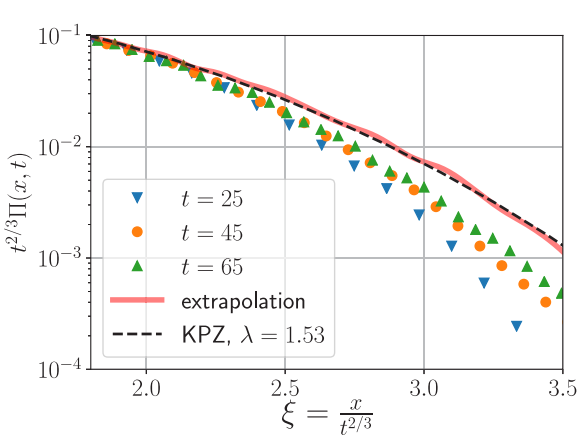

(b)

FIG. 11. (a) Numerical results for $\Delta x(t)$ divided by the leading power law $t^{2 / 3}$. Different scenarios for the correction term are shown: $t^{-1 / 2}$ (left) and $t^{-1 / 3}$ [right, Fig. 6(c) is duplicated here for easier comparison]. (b) Same data as in Fig. 7(a) with a different extrapolation (red line), which is consistent with the $t^{-1 / 2}$ correction.

where $\mathcal{L}_{x, x+1}$ acts on sites $x, x+1$ only. Then, $\mathcal{U}(\Delta t)$ can be subjected to a Suzuki-Trotter decomposition, e.g., of second order

$$
\begin{aligned}
\mathcal{U}(\Delta t)= & e^{-i \mathcal{L}_{1,2} \Delta t / 2} \ldots e^{-i \mathcal{L}_{L-1, L} \Delta t / 2} e^{-i \mathcal{L}_{L-1, L} \Delta t / 2} \\
& \ldots e^{-i \mathcal{L}_{1,2} \Delta t / 2}+O\left(\Delta t^{3}\right)
\end{aligned}
$$

as used in this work. Truncation (in terms of singular values) is carried out simultaneously after each bond update in order to keep the bond dimensions below $\chi_{\max }$ (simply denoted by $\chi$ throughout this work). The corresponding error is referred to as "truncation error." Throughout this work, we choose a very small cutoff for the singular values in the truncation procedure. Therefore, the maximum bond dimension alone controls the matrix product approximation in our simulations.

\section{Convergence}

In Fig. 9 we show additional data illustrating the dependence of the numerical results on $\chi$ as well as the Trotter time increment $\Delta t$. Choosing a smaller $\Delta t$ will decrease the error due to the Trotter decomposition of the time-evolution operator. On the other hand, choosing a smaller value of $\Delta t$ requires a larger number of truncations to be carried out within a given window of time. Therefore, data obtained using a smaller $\Delta t$ are not necessarily more accurate. Furthermore, as soon as the results are not strictly converged with respect to $\chi$ [as is the case for the longest times shown in, e.g., Fig. 6(b)], a dependence on the precise value of $\Delta t$ is also expected. However, we demonstrate in Fig. 9 that a certain degree of stability with respect to varying $\Delta t$ can be observed.

In accordance with previous works [21,25], we observe that truncation errors generally introduce unphysical fluctuations in $\Pi(x, t)$. Those are most pronounced near $x=0$ while the fluctuations in the tails appear only at longer times in the form of more regular oscillations [see Fig. 9(b)].

\section{APPENDIX B: DIFFUSION CONSTANT AT $\Delta=\mathbf{2}$}

For anisotropy $\Delta>1$, the spin dynamics at vanishing magnetization $m=0$ is known to be normal diffusive [8]. In the long-time limit, it is therefore expected that $\partial_{t} \Delta x^{2}(t) \stackrel{t \rightarrow \infty}{=}$ $2 D t$ with $D$ denoting the diffusion constant. The results shown in Fig. 10 demonstrate that this long-time limit cannot be reached reliably with bond dimensions $\chi<2000$. A naive lower bound $D \gtrsim 0.63$ is obtained from these data by taking the maximum value reached for the largest bond dimension $\chi=1600$ available. This value should be contrasted with the value $D \approx 0.4$ shown in Ref. [17] [cf. Fig. 2(b), inset, of that reference], which employed the same protocol for simulating spin dynamics albeit with a much smaller bond dimension of $\chi=200$. Our result is consistent with earlier works evaluating the diffusion constant by means of a direct evaluation of the current-current correlator at high temperature. In particular, for $\Delta=2$, a lower bound of $D \gtrsim 0.56$ was given in Ref. [42], based on numerical data for $t \leqslant 17$. Recently, Ref. [8] obtained an analytic result of $D \approx 0.77$ and they give a numerical estimate of $D \approx 0.73$, which was obtained by an extrapolation scheme with respect to time. It is shown in Fig. 10 that, applying the same extrapolation scheme, our data appear consistent with a very similar value of $D$.

\section{APPENDIX C: CORRECTIONS TO SCALING: FURTHER DISCUSSION AND ALTERNATIVE SCENARIO}

In Sec. III B 2, we found that the numerical data for the spin correlation function can be described by $\Pi(x, t)=$ $\left.\Pi_{\mathrm{KPZ}}^{\lambda}(\xi)\left[1+g(\xi) t^{-y}\right]\right)$ with $y=\frac{1}{3}$ and $\lambda=1.57$. For comparison, we show an alternative scenario in Fig. 11 corresponding to $y=\frac{1}{2}$ and $\lambda=1.53$, which also allows for consistent longtime extrapolations of $\Delta x(t)$ and $\Pi(x, t)$. However, the $t^{-1 / 3}$ correction appears to describe the time dependence of $\Delta x(t)$ more accurately down to very short times.
[1] M. Takahashi, Thermodynamics of One-Dimensional Solvable Models (Cambridge University Press, Cambridge, 1999).
[2] O. A. Castro-Alvaredo, B. Doyon, and T. Yoshimura, Phys. Rev. X 6, 041065 (2016). 
[3] B. Bertini, M. Collura, J. De Nardis, and M. Fagotti, Phys. Rev. Lett. 117, 207201 (2016).

[4] V. B. Bulchandani, R. Vasseur, C. Karrasch, and J. E. Moore, Phys. Rev. B 97, 045407 (2018).

[5] B. Doyon, T. Yoshimura, and J.-S. Caux, Phys. Rev. Lett. 120, 045301 (2018).

[6] J. De Nardis, D. Bernard, and B. Doyon, Phys. Rev. Lett. 121, 160603 (2018).

[7] S. Gopalakrishnan, D. A. Huse, V. Khemani, and R. Vasseur, Phys. Rev. B 98, 220303(R) (2018).

[8] J. De Nardis, D. Bernard, and B. Doyon, SciPost Phys. 6, 049 (2019).

[9] B. Doyon, J. Dubail, R. Konik, and T. Yoshimura, Phys. Rev. Lett. 119, 195301 (2017).

[10] M. Fagotti, Phys. Rev. B 96, 220302(R) (2017).

[11] M. Collura, A. De Luca, and J. Viti, Phys. Rev. B 97, 081111(R) (2018).

[12] E. Ilievski, J. De Nardis, M. Medenjak, and T. Prosen, Phys. Rev. Lett. 121, 230602 (2018).

[13] K. Fabricius and B. M. McCoy, Phys. Rev. B 57, 8340 (1998).

[14] J. M. P. Carmelo and T. Prosen, Nucl. Phys. B 914, 62 (2017).

[15] J. M. P. Carmelo, T. Prosen, and D. K. Campbell, Phys. Rev. B 92, 165133 (2015).

[16] M. Žnidarič, J. Stat. Mech: Theory Exp. (2011) P12008.

[17] M. Ljubotina, M. Žnidarič, and T. Prosen, Nat. Commun. 8, 16117 (2017).

[18] J. Richter and R. Steinigeweg, Phys. Rev. B 99, 094419 (2019).

[19] S. Gopalakrishnan and R. Vasseur, Phys. Rev. Lett. 122, 127202 (2019).

[20] M. Dupont and J. E. Moore, arXiv:1907.12115.

[21] M. Ljubotina, M. Žnidarič, and T. Prosen, Phys. Rev. Lett. 122, 210602 (2019).

[22] E. M. Stoudenmire and S. R. White, ITensor - Intelligent Tensor Library, http://itensor.org.

[23] T. Barthel, New J. Phys. 15, 073010 (2013).

[24] D. M. Kennes and C. Karrasch, Comput. Phys. Commun. 200, 37 (2016).

[25] S. Gopalakrishnan, R. Vasseur, and B. Ware, Proc. Natl. Acad. Sci. USA 116, 16250 (2019).
[26] V. Hunyadi, Z. Rácz, and L. Sasvári, Phys. Rev. E 69, 066103 (2004).

[27] V. Eisler and Z. Rácz, Phys. Rev. Lett. 110, 060602 (2013).

[28] V. B. Bulchandani and C. Karrasch, Phys. Rev. B 99, 121410(R) (2019).

[29] M. Kardar, G. Parisi, and Y.-C. Zhang, Phys. Rev. Lett. 56, 889 (1986).

[30] H. van Beijeren, Phys. Rev. Lett. 108, 180601 (2012).

[31] M. Kulkarni and A. Lamacraft, Phys. Rev. A 88, 021603(R) (2013).

[32] M. Arzamasovs, F. Bovo, and D. M. Gangardt, Phys. Rev. Lett. 112, 170602 (2014).

[33] I. Poboiko and M. Feigel'man, Phys. Rev. B 94, 195420 (2016).

[34] R. Samanta, I. V. Protopopov, A. D. Mirlin, and D. B. Gutman, Phys. Rev. Lett. 122, 206801 (2019).

[35] M. Prähofer and H. Spohn, J. Stat. Phys. 115, 255 (2004).

[36] M. Prähofer and H. Spohn, Exact scaling functions for onedimensional stationary KPZ growth, http://www-m5.ma.tum. de/KPZ.

[37] A. Das, M. Kulkarni, H. Spohn, and A. Dhar, Phys. Rev. E 100, 042116 (2019).

[38] J. De Nardis, M. Medenjak, C. Karrasch, and E. Ilievski, Phys. Rev. Lett. 123, 186601 (2019).

[39] U. Schollwöck, Ann. Phys. 326, 96 (2011), January 2011 special issue.

[40] S. Paeckel, T. Köhler, A. Swoboda, S. R. Manmana, U. Schollwöck, and C. Hubig, Ann. Phys. 411, 167998 (2019).

[41] M. Zwolak and G. Vidal, Phys. Rev. Lett. 93, 207205 (2004).

[42] C. Karrasch, J. E. Moore, and F. Heidrich-Meisner, Phys. Rev. B 89, 075139 (2014).

[43] A. G. Abanov and P. B. Wiegmann, Phys. Rev. Lett. 95, 076402 (2005).

[44] I. V. Protopopov, D. B. Gutman, P. Schmitteckert, and A. D. Mirlin, Phys. Rev. B 87, 045112 (2013).

[45] J. Richter, N. Casper, W. Brenig, and R. Steinigeweg, Phys. Rev. B 100, 144423 (2019).

[46] C. B. Mendl and H. Spohn, J. Stat. Mech (2015) P08028.

[47] M. Kulkarni, D. A. Huse, and H. Spohn, Phys. Rev. A 92, 043612 (2015). 